Revue européenne des migrations internationales

vol. $19-n^{\circ} 2$ | 2003

Les initiatives de l'étranger et les nouveaux

cosmopolitismes

\title{
Les recompositions d'une centralité commerçante immigrée : la Place du Pont à Lyon
}

\section{Alain Battegay}

\section{(2) OpenEdition}

\section{Journals}

Édition électronique

URL : https://journals.openedition.org/remi/432

DOI : $10.4000 /$ remi.432

ISSN : $1777-5418$

Éditeur

Université de Poitiers

Édition imprimée

Date de publication : 1 juillet 2003

Pagination : 9-22

ISBN : 2-911627-34-2

ISSN : 0765-0752

Référence électronique

Alain Battegay, «Les recompositions d'une centralité commerçante immigrée : la Place du Pont à Lyon », Revue européenne des migrations internationales [En ligne], vol. 19 - n² 2 2003, mis en ligne le 19 avril 2007, consulté le 14 avril 2022. URL : http://journals.openedition.org/remi/432 ; DOI : https:// doi.org/10.4000/remi.432

Ce document a été généré automatiquement le 14 avril 2022

(c) Université de Poitiers 


\title{
Les recompositions d'une centralité commerçante immigrée : la Place du Pont à Lyon
}

\author{
Alain Battegay
}

1 Depuis plusieurs années, des recherches sur les migrations développent des approches centrées sur le couple mobilité/territoire, et insistent sur les mobilités des migrants et leurs circulations migratoires. Elles incitent à porter attention à la manière dont ces mobilités mettent en interaction des espaces discontinus et dessinent des routes géographiques et sociales entre régions, villes d'origines, d'installation et de passage. Elles montrent que ces routes s'articulent dans les villes et les agglomérations européennes et notamment françaises, avec des ancrages sociaux et spatiaux constitués par les migrations, et donnent lieu à des carrefours où se croisent différents réseaux migratoires. Elles signalent que ces carrefours d'informations et de transactions prennent souvent l'allure de places commerçantes et d'affaires, qui exercent des fonctions de centralité dans l'approvisionnement de populations en situation migratoire résidant dans les régions concernées. Différentes recherches font ainsi apparaître des configurations territoriales originales, transnationales, avec des géographies et des topologies particulières, coordonnant mobilité et sédentarité, articulant ancrages, passages et voisinages sur des registres tant économiques que sociaux et culturels.

Des centralités immigrées

2 Au carrefour logistique, économique et social, de ces configurations territoriales, le rôle particulier de certains centres-villes a été souligné dans différentes villes françaises (La Goutte d'Or, Barbès, le XIII ${ }^{e}$ arrondissement, Belleville à Paris, Belsunce à Marseille, La Place du Pont à Lyon). L'existence et l'activité de ces «centralités immigrées » est attestée dans les grandes villes de l'hexagone, pour certaines depuis plus d'un demisiècle : elles ont des histoires, des positionnements urbains, des modes de formation différents, particuliers à chaque ville et qui renvoient à des configurations et des histoires migratoires singulières. Depuis quelques années, plusieurs d'entre elles se 
recomposent et se redéploient, en lien avec les réorientations des circulations migratoires dont elles relèvent et avec l'apparition d'autres places marchandes qui font sens à l'échelle des villes, des agglomérations, des aires urbaines dans lesquelles elles s'inscrivent (par exemple le Marché du soleil et le Marché aux puces à Marseille, Marché de Vintimille). Leur observation a fait apparaitre des caractéristiques communes: ces espaces fonctionnent à la fois comme des places et des pôles, non seulement comme des quartiers populaires et composites de grandes villes mais aussi comme des carrefours de mobilités et d'informations, des opérateurs de circulation économique et culturelle. En ces lieux convergent des dispositifs réticulaires qui se déploient à différentes échelles territoriales et donnent à ces quartiers des caractères de centralités alors même que localement, les morceaux de ville qu'ils forment apparaissent parfois comme des espaces restreints et spécifiques. En outre ils ne prennent pas la forme d'un territoire compact mais d'une agrégation de micro-lieux entretenant entre eux des rapports de complémentarité et de concurrence, et générant localement des effets d'attraction en tant que centre d'achats pour des clientèles et des usagers proches par leurs origines nationales, participant du même univers d'approvisionnement (particuliers et professionnels, ménages et entreprises) et résidant dans l'agglomération et au-delà, alors que d'autres populations (y compris les autochtones) les fréquentent pour accéder à des lieux « exotiques».

3 Certaines de ces centralités, dont la formation renvoie en partie à des moments de déshérence urbaine, font aujourd'hui l'objet de recompositions. Les limites spatiales, les fonctions urbaines et d'agglomération, les peuplements de ces morceaux de ville se redéfinissent : leurs redéfinitions, liées à des enjeux locaux, participent de processus qui touchent aussi d'autres quartiers anciens de centre ville, mis à l'épreuve de l'étalement des agglomérations, de la restructuration du commerce urbain et d'usages culturels et patrimoniaux à l'échelle des aires urbaines. Au-delà, les recompositions de ces centralités immigrées sont aussi liées à des conjonctures et des opportunités (par exemple possibilités d'obtention de visas, situations économiques et sécuritaires dans le pays d'origine) dont dépendent leurs fonctionnements comme lieux de circulations de populations, d'informations, de marchandises et comme lieux de croisement et de construction de réseaux sociaux (familiaux, migratoires, commerciaux et d'affaires). Localement, elles sont loin d'être valorisées par les villes dans lesquelles elles prennent place, et leurs recompositions sont orientées par les contraintes et limitations qui sont imposées de l'extérieur par les autorités et les sociétés urbaines à leur maintien et à leur développement. De l'intérieur, ces centralités, qui ne peuvent plus compter sur leurs seuls bénéfices en tant que niche pour attirer des clientèles dont les univers d'approvisionnement se diversifient, redéfinissent également leur offre en renégociant dans leurs principes de fonctionnement, les liens entre normes d'appartenance et normes marchandes, entre normes de confiance et normes de qualité, entre valeur traditionnelle et valeur d'innovation.

4 C'est dans cette perspective que nous revisitons la Place du Pont à Lyon, qui, à l'échelle de l'agglomération lyonnaise, figure une de ces centralités commerçantes immigrées ${ }^{1}$. La place du pont : espaces et monde

5 Dans l'agglomération lyonnaise, le quartier de la Place du pont hérite d'une histoire tissée par des vagues de migrations successives ou concomitantes qui ont trouvé, à partir de la fin du xix siècle, dans l'ancien faubourg de la rive gauche du Rhône (commune indépendante jusqu'en 1852), des opportunités singulières d'installation. La 
présence d'Italiens et de Grecs est attestée dans le quartier au début du Xx $x^{e}$ siècle, alors que dans les années 1920-1930 des Algériens sont fortement présents dans le quartier. Dans les années 1960-1970, la Place devient un haut lieu d'approvisionnement des populations maghrébines, en lien avec l'implantation de commerçants juifs venant du Maroc et d'Algérie.

6 Étudiée dans les années 1980, la Place du Pont apparaissait comme une centralité d'approvisionnement pour des populations maghrébines de l'ensemble de l'agglomération lyonnaise. Des études concernant les successions commerçantes confirmaient alors le déclin progressif des commerces «français » et signalaient un départ partiel des commerces tenus par les Juifs: elle montraient que ce départ ne pouvait pas se comprendre à partir des seules interactions locales, mais qu'il était lié aux redistributions des populations juives dans l'agglomération, que ce soit dans l'activité commerçante de la ville, dans ses implantations résidentielles, dans ses lieux communautaires. D'autres observations montraient que des bazaris marocains s'installaient à proximité de la Place, selon des stratégies différenciées qui conjuguaient dans leur parcours d'accès des liens d'originaires, des alliances commerciales, des relations d'apprentissage, d'entraide et de concurrence.

7 Depuis lors, les transformations de la Place ont été nombreuses: des opérations de rénovation conduites dans la perspective d'une percée urbaine ont à ce point marqué le quartier, au cours des années 1990, que l'idée de la fin probable de la Place comme centralité commerçante immigrée était un sentiment partagé. Le bâti a été profondément modifié : les opérations inachevées de rénovation urbaine ont conduit à la destruction d'immeubles réputés insalubres. Elles ont flanqué la façade du quartier d'un grand immeuble moderne (le CLIP) après la destruction d'un immeuble haussmanien dédié à un magasin à grande surface. Le métro a rendu plus accessible le quartier après un chantier de plusieurs mois, et le tramway a été mis en service en 2002. Une place publique a été aménagée là où des immeubles insalubres avaient été détruits, les terrains étant transformés en parkings provisoires, et des travaux de réaménagements des sorties de métro sont en cours au pied du CLIP. Les commerces de proximité qui jouent sur les clientèles de quartier ont décliné : des moyennes surfaces affichant des enseignes de la grande distribution se sont installées en bordure de la Place et ont repris cette fonction, tandis que les spécialités des commerces se sont recomposées en lien avec les demandes de clientèles d'origine maghrébine provenant de l'ensemble de l'aire urbaine et que, dans certaines rues, des locaux commerciaux vides cherchent repreneurs.

8 Cette évocation de la Place qui la décrit à partir d'un espace bâti, formant une sorte de triangle aux contours imprécis, ne compte aujourd'hui guère plus, selon les estimations et les limites retenues, qu'entre 2000 et 5000 habitants. C'est donc un quartier de peu de poids, s'il est rapporté à l'échelle d'une agglomération qui compte plus d'un million et demi d'habitants.

9 Mais la Place du Pont n'est pas réellement un quartier et sa grandeur ne peut s'évaluer à l'aune de ces métriques. C'est d'abord dans l'actualité urbaine un morceau de ville qui a fait parlé de lui, en raison d'opérations de rénovation urbaine qui n'ont pas connu les succès escomptés: les grands axes qui devaient être percés auraient du détruire l'essentiel du tissu urbain antérieur, avec la construction d'un immeuble pensé comme un geste architectural devant faire repère et marquer l'implication de ce morceau de ville dans une modernité urbaine, limitant son marquage maghrébin à une touche 
d'exotisme méditerranéen. L'immeuble a vu le jour, avec cependant moins d'envergure qu'initialement prévu et l'opération achevée n'est pas (encore ?) allée à son terme : la Place du Pont est encore un lieu actif dont le nom fait sens, alors qu'il était lui aussi promis à restructuration.

C'est aussi, dans le même temps le nom d'une centralité immigrée qui s'articule à l'espace local sans s'y fondre et qui se présente différemment selon ceux qui en parlent. Les autorités municipales et les dispositifs de gestion découpent le tissu urbain local selon des critères qui s'indexent à leurs périmètres de compétences et d'interventions : «la Place du Pont» leur apparaît une dénomination aux contours flous qui leur convient mal et ils préfèrent des désignations plus descriptives, plus précises, qui leur permettent des tracés plus nets. Les géographies de la Place que donnent les gens du Maghreb sont plus fluides et se réfèrent à un espace de ressources et d'approvisionnements. Elles se décrivent en lignes commerçantes et en emplacements plutôt qu'en blocs compacts, en moments autant qu'en espaces, en repères plutôt qu'en contiguïtés territoriales. Si localement c'est le morceau de ville sous tutelle administrative, politique et urbaine qui fait officialité, dans les langages vernaculaires, le terme "Place du Pont» est largement connu, dans le quartier, à l'échelle de l'agglomération, dans les mémoires de migrations de la ville et de la région, mais aussi au-delà, parmi les gens du Maghreb à Marseille et à Paris, et au Maghreb même, notamment dans l'Est Algérien. Il fait sens, là-bas, même pour des gens qui ne sont jamais venus en France, et qui ignorent tout autre aspect de Lyon. De ce point de vue, la Place du Pont est un lieu urbain et un temps de la ville plutôt qu'un quartier, un espace de ressources plutôt qu'un espace de proximité, un moment dans des parcours et un repère dans des circulations migratoires plutôt qu'une unité de voisinage résidentiel.

11 Revisiter ce lieu, c'est d'abord prendre acte de ces déficits et différentiels de reconnaissance, de cette double lecture qui le valorise et le dévalorise dans des mouvements concomitants et des usages simultanés. C'est ensuite interroger à double titre son destin, marqué par cette superposition dans les mêmes espaces de territoires dont les significations et les syntaxes sociales s'interconnectent et se dissocient. C'est encore prendre la mesure du temps, et suggérer que ce morceau de ville qui avait joué à partir des années 1930, et plus fortement encore dans l'après-guerre jusqu'aux années 1970, le rôle de quartier de première implantation et de porte d'entrée dans la ville pour des immigrants de l'intérieur (comme les Ardéchois) ou d'au-delà des frontières (Italiens, Juifs d'Europe de l'Est et du Maghreb, Algériens, Tunisiens, Marocains), a su en développant sa fonction marchande, se donner d'autres rôles dans les parcours de migrants et dans l'agglomération lyonnaise.

12 C'est sur le fait que les configurations d'usages de la Place du Pont se sont transformées, en lien avec des transformations contemporaines des pratiques urbaines des migrants que nous voudrions ici mettre l'accent.

«Les hommes debout » : un monde et un moment de la place

$13 \mathrm{Au}$ regard de la presse et des autorités locales, la Place est d'abord un quartier louche : un quartier de deal, où les revendeurs travaillent dans les rues, de manière particulièrement visibles, et dont les transactions se concentrent ou s'étalent autour du quartier, tenant compte de l'accessibilité réduite et des pressions policières. C'est aussi un quartier où des " hommes debout » se regroupent au pied du "bâtiment moderne ", en dépit du mobilier urbain destiné à provoquer l'inconfort des rencontres. Ces regroupements reprennent une ancienne tradition datant de plus d'un quart de siècle, 
d'un ensemble de pratiques articulées et difficilement dissociables, de rencontres, de sociabilité et d'informations à l'échelle de la ville. Plusieurs de nos interlocuteurs nous ont ainsi raconté que c'est là qu'ils avaient réussi, lors de l'éboulement d'une partie d'Alger en novembre 2001, à avoir des nouvelles de leurs familles alors que toutes les lignes téléphoniques étaient coupées, grâce à des rencontres. D'autres viennent chercher ici des informations pour de bonnes affaires: lots à acheter, voitures d'occasion, fonds de commerce. D'autres encore, et parfois les mêmes, viennent pour changer de l'argent (plusieurs de nos interlocuteurs parlent en plaisantant de ce regroupement comme de «Wall Street » : «si tu veux savoir le cours de l'or et du dollar, il n'y a pas mieux pour se renseigner »). Certains mentionnent le petit marché aux puces informel des « hommes debout » où s'étalent des objets disparates et sans grande valeur (vêtements d'occasion, montres, ustensiles de maison), et où se pratique de la revente de matériel (portables...) dont la provenance est incertaine. D'autres encore viennent chercher des ressources (des plans, du travail, des adresses) alors qu'ils sont en situation d'urgence et de précarité, et d'autres encore passent par le lieu des « hommes debout », une des portes du quartier, en venant chercher «à la Place » de la drogue. Ces regroupements plurifonctionnels dessinent en tout cas un monde d'hommes que de nombreuses femmes disent éviter ou contourner lorsqu'elles accèdent au quartier, alors que d'autres traversent ces regroupements sans encombre, comme en témoignent les observations de cette commerçante d'un magasin riverain qui organise des cérémonies de mariage et dont la clientèle est aussi composée de femmes et de familles. L'organisation sociale de cet espace est plus complexe qu'il n'y paraît : des observations montrent comment des anciens de l'immigration en ont fait un lieu d'activités et de présence relativement régulière alors que les lieux de rencontres qu'ils fréquentaient à proximité de leurs habitations se sont défaits, les anciens n'étant plus là ou les cafés ayant disparus; elles montrent également la fonction « repère » pour ceux qui viennent d'arriver parfois dans l'urgence ou dans des situations de clandestinité, à la recherche de relations et de ressources, de travail, de papiers; elles montrent encore comment des populations de l'Est européen, identifiées sous le nom de "roumains " s'y retrouvent et utilisent parfois ces moments et ces espaces de regroupement comme une occasion de marché, des fourgonnettes apportant des vêtements et des objets destinés à la vente. Ces différents usages qui participent à la permanence de cet espace-temps en font aussi un lieu d'intrigue qui alimente des tensions et des rumeurs : tel commerçant riverain lors d'une réunion publique a porté haut et fort la plainte de l'insécurité et de la gêne provoquée par les «hommes debout "; d'autres tentent de dissocier, parmi ces hommes, les anciens de l'immigration qui font partie de la Place même s'ils n'y habitent pas, des étrangers qui viennent d'ailleurs et font de la Place un lieu de rassemblement et de trafic qui nuit à son image et à la réputation de tout le quartier. Des habitants soulignent le climat de tension qui prévaut parfois, faisant autant référence à la présence policière qui effectue des contrôles, de plus en plus fréquents et visibles durant les campagnes électorales, qu'à des remarques et des gestes des occupants de la Place, qui les insécurisent sans les viser. Ceux qui fréquentent le quartier occasionnellement émettent des avis partagés : certains trouvent que les « hommes debout » font «tache » et qu'ils nuisent à l'image $\mathrm{du}$ quartier, tandis que d'autres sont positivement intrigués par l'animation du lieu alors que la ville est déserte le dimanche après-midi ; d'autres encore remarquent, en le déplorant ou en s'en félicitant, que cette présence marque l'échec de l'opération de " rénovation-nettoyage » du quartier, et certains soulignent, avec admiration, la force 
des habitudes et la ritualisation de cet espace de rencontres, qui persistent en dépit de toutes les tentatives, urbanistiques et policières qui visent à déloger les «hommes debout ». Les lignes de partage de ces appréciations entremêlées ne permettent pas de rapporter ces avis au fait d'habiter le quartier, d'y tenir commerce ou de venir s'y approvisionner, au fait d'être d'origine maghrébine ou de ne pas l'être, au fait d'être une femme ou un homme, un jeune ou un vieux. Mais, à la conjonction de ces avis, « les hommes debout " s'imposent comme une des figures de la Place, ils font partie du paysage et des mondes de la Place, ils en forment un des mondes, une des images, un des emblèmes, une des ambiances, manifestant là qu'il y a porosité entre accessibilité réservée et fréquentation publique, entre illégal et illégitime, entre interconnaissance et inter-reconnaissance.

Recompositions commerciales et recompositions d'usages

Nous avons orienté nos investigations sur l'offre commerçante de la Place pour comprendre les ressources qu'elles procurent ou qu'elles génèrent, les demandes auxquelles elles s'ajustent. À travers ces lunettes, plusieurs phénomènes marquants apparaissent : d'une part, la proximité devient un mode mineur d'usage de la Place, et le nombre de commerces exclusivement de proximité tend à se réduire; d'autre part, les successions commerçantes montrent que le triangle de la Place tend à se spécialiser avec l'arrivée de nouveaux commerçants du Maghreb (Algériens, Tunisiens, Marocains) et le déclin ou le départ de commerces alimentaires tenus par des «Français » et des "Juifs ", souvent originaires d'Afrique du Nord. Les commerces "s'arabisent» disent des anciens de la Place qui le constatent sans forcément le déplorer. Enfin les activités de la Place s'organisent selon des calendriers indexés à des rythmes sociaux, des styles de vie, des pratiques particulières : la Place du Pont est un des rares espaces de la ville commercialement actif le dimanche, et sa fréquentation est particulièrement dense lors des fêtes religieuses (Ramadan ou l'Aïd), des mariages et des périodes estivales ponctuées par des déplacements vers les pays d'origine et des approvisionnements conséquents.

15 Ces éléments signalent une réorganisation du dispositif commercial de la Place qui adapte son offre à des usages qui ne sont pas majoritairement de proximité ni de quotidienneté, et à des rythmes sociaux spécifiques à certaines populations. C'est peutêtre là une manière de comprendre la résistance de la Place à la fois aux nombreux travaux qui l'ont enclavé spatialement en réduisant son accessibilité physique, et à la restructuration du commerce urbain avec l'apparition de centres commerciaux et de marchés fréquentés aussi par ceux qui viennent s'approvisionner à la Place. Elle aurait résisté à ses contextes et trouvé de nouvelles pertinences en réaménageant son offre vers des produits et des services commercialement « résiduels » mais à haute valeur sociale et symbolique, non prise en compte par d'autres appareils de distribution.

C'est ce que semble confirmer l'analyse des spécialités commerçantes et des types de service à la mobilité. Ces derniers sont ici particulièrement denses : agences de voyages, de cars, bureaux d'import-export d'automobiles, agences téléphoniques à la carte. Cette concentration d'offres de biens et de services en lien direct avec des déplacements s'articule à des offres de biens matériels et symboliques dont la valeur, on l'a déjà mentionnée, est liée à des styles de consommation, à des types de calendriers, à des rituels spécifiques: pour les populations au regard desquelles ces biens font sens (boutiques liées au mariage, aux fêtes, aux cérémonies, pompes funèbres musulmanes, concentration de boucheries halal, librairies musulmanes), cet espace particulier 
concentre des ressources non seulement pour les déplacements mais également pour une mobilité culturelle "sur place", articulant des affirmations minoritaires et des établissements dans l'aire urbaine.

Nous avons ainsi porté un regard plus attentif sur des mobilités d'approvisionnement liées au mariage et sur la concentration de nombreux magasins proposant des robes de fêtes et de cérémonies (plus de 25 boutiques de ce genre) sans compter nombre d'autres magasins qui proposent des articles de bijouterie, des textiles, des couvertures, des parures de bains, des tapis, des services à vaisselle et à cafés, des fruits secs (amandes, pistaches, pruneaux pignons). Un premier repérage des implantations spatiales montre que ces boutiques de robes de fête et de mariage se répartissent de part et d'autre d'une rue commerçante, la rue Paul Bert, sorte de couture et de frontière urbaine : dans la rue elle-même, voisinent magasins de robes de style oriental et magasins de robes de mariées «blanches ». Mais ces magasins se déploient séparément de part et d'autre de la rue, dans le tissu urbain local. Cet ordre spatial de l'offre ne se traduit pas mécaniquement dans les parcours des clientèles : les boutiques de robes de mariées qui apparaissent hors des limites du "quartier arabe ", dans les grands boulevards et avenues qui forment des axes commerçants réputés frontières urbaines majeures de la Place du Pont, attirent aussi des familles maghrébines qui achètent des robes de mariées blanches pour le trousseau de la mariée (une des sept robes habituellement portées). C'est aussi cette labilité de la Place, lisible à partir des pratiques d'approvisionnement alors qu'elle est inscrite dans un ordre territorial local pourtant fortement structuré, qui nous fait comprendre un des principes de sa consistance, une manière de déborder, d'étaler, de trouver des complémentarités d'usages.

Un autre mouvement actuel de redéploiement de la Place comme ressource mérite aussi d'être signalé : l'apparition, depuis 4 ou 5 ans, de boutiques spécialisées dans l'organisation de mariages, proposant à la carte ou au menu un ensemble de services (réservation de salles, location de robes de mariées et d'accessoires, de tenues vestimentaires, produits de traiteur, et henné). Les clients de ces magasins d'un type nouveau viennent de toute la région et notamment des petites villes (Vienne, Clermont, Saint-Étienne), dans lesquelles ces services n'existent pas. Parmi les services proposés, dont le format est probablement importé du Maroc, ceux de la Négafa semblent particulièrement significatifs. La Négafa étant une femme chargée de préparer la mariée, de la maquiller, de l'aider lors du changement des sept tenues traditionnelles, et d'effectuer le tatouage au henné des mains. Or, il s'agit là d'actes qui sont, dans les habitudes algériennes, habituellement assurés par les membres de la famille et considérés comme trop chargés de signification pour être confié à un étranger.

Le constat d'une marchandisation de pratiques, qui étaient autrefois domaine réservé des proches, nous a alors conduit à porter notre attention sur l'apparition dans le dispositif commercial de la Place du Pont d'autres services commercialisés témoignant du même mouvement. Ainsi en est-il des magasins de pompes funèbres musulmanes alors même que le rapatriement des corps était auparavant l'affaire, pour les populations maghrébines de France, des Amicales, des réseaux consulaires, des familles et des solidarités d'originaires en migration. Ils offrent des formules diversifiées d'assurance-décès et d'enterrement, allant de l'enterrement sur place dans un carré musulman au rapatriement du corps avec un billet d'accompagnateur. Ces indications tendraient à appuyer l'hypothèse que la Place fonctionnerait ici à la fois comme un lieu de mise en marché de services à destination de populations maghrébines et un lieu de 
mise en concurrence de services auparavant assurés par des réseaux familiaux ou d'originaires, ou qui étaient réservés à des monopoles « communautaires ». En ce sens, «la Place» serait aussi un lieu d'innovation prenant appui sur des pratiques « traditionnelles » tout en les transformant.

D'autres types d'offres commerciales ressortent de la même logique. Ainsi en est-il de la concentration et des recompositions des boucheries musulmanes de la Place qui renvoient non seulement à des dimensions locales d'opportunités d'établissement mais à des transformations de la filière halal elle-même: le marché de la viande halal implique depuis plusieurs années de nouvelles configurations d'acteurs et s'élargit en s'adaptant à de nouveaux styles de consommation et de nouveaux types de clientèles. Depuis le début des années 1990, le label halal est devenu, en France et à Lyon en particulier, un des procédés majeurs pour convaincre le consommateur de l'authenticité du produit et un enjeu de crédibilité pour des réseaux musulmans qui investissent le domaine dans une action tant sociale que culturelle. Les opérateurs halal en lien avec des bouchers donnent à leurs entreprises commerciales l'aspect d'entreprises morales : leurs initiatives valent aussi comme des tentatives d'implanter des ressources symboliques dans un contexte d'individuation de la foi, où se mettent en place de nouvelles habitudes de consommation qu'ils entendent favoriser et renforcer et auxquelles ils doivent s'ajuster. C'est ainsi qu'ils font de la Place du Pont une vitrine, un espace de captations de clientèle, d'épreuve de leur réputation et de leur crédibilité, et de tests de leurs produits, une réserve de sens et un espace d'expérimentation.

De même l'apparition et l'augmentation significative des librairies musulmanes au cours de ces dernières années vont dans le même sens. Alors que l'espace de l'écrit était réduit, on peut aujourd'hui compter six libraires où l'on peut trouver des livres et des cassettes, vidéo et audio, en français et en arabe, se rapportant à l'Islam, ainsi que des objets liés aux pratiques religieuses et à l'exercice du culte, ou des vêtements affichant une singularité musulmane. Articulés sur des réseaux de musulmans, et parfois euxmêmes éditeurs de livres, brochures et cassettes, ces magasins proposent des produits qui composent une offre adaptée à des pratiques ritualistes de l'Islam comme à des approches intellectualisées, et qui prennent en compte les processus d'individuation de la foi qui marque les rapports contemporains des musulmans à l'Islam.

La place du pont, espace urbain de ritualités minoritaires

C'est dans cette logique que se manifesterait le nouveau rôle de la Place du Pont: espace de ressources pour des populations dont les manières de vivre en migration se diversifient et se recomposent, et espace vitrine, où s'exposent et se mettent à l'épreuve de nouveaux produits et de nouveaux services. Dans ces évolutions et ces transformations, les rapports qualité/prix et les normes de qualité semblent gagner du terrain : si les bénéfices d'un effet de niche et d'exclusivité sont toujours sensibles, ils ne semblent plus suffire à attirer des consommateurs dont les choix en matière d'approvisionnement se diversifient, dont les exigences en matière de qualité et de nouveauté s'affirment, et qui ne font plus jouer des normes d'appartenance comme seuls critères d'approvisionnement. Loin de ne valoir qu'en tant que lieu conservatoire, marché louche de la survie et de l'illicite, îlot de captivité résidentielle de certains de ses habitants, cette centralité « immigrée » se révèle un agencement social et territorial non seulement d'accompagnement mais de transformations des pratiques de populations en situation migratoire. 
Cette approche des offres commerçantes de la Place et de leurs transformations récentes montre qu'elles se redéploient en s'ajustant à des demandes qu'elles contribuent à orienter : leur attractivité vient de leur pertinence aux regards de leurs clientèles qui les considèrent comme des ressources pour des «mobilités culturelles » agissant sur place autant que dans des dimensions transnationales.

C'est ce qui ferait que l'existence de ce morceau de ville ne serait pas résiduelle, témoignant de l'inachèvement de processus d'intégration de populations destinées à se mouler dans les manières communes de vivre les villes au point d'en devenir invisibles, ou des limites d'opérations de rénovation urbaine tendant à modifier la composition de ces lieux. Même si sa pérennité dans son espace actuel est en partie incertaine, notamment en raison d'opérations de reconquête urbaine toujours en passe d'être activées et aussi en raison de la restructuration des commerces urbains, la Place du Pont n'est pas qu'un lieu de mémoire de l'immigration dans la ville : elle est aussi porteuse de dynamiques contemporaines. Même si elle est traitée comme dérangeante ou indésirable, les fonctionnements auto-produits qui s'y développent témoignent en effet de leur pertinence comme centres de ressources adaptés à des populations spécifiques qui en font des usages « intercalaires », venant chercher ici ce qu'elles ne trouvent pas ailleurs (ambiance, relations, produits, services) alors même qu'elles fréquentent et qu'elles participent à d'autres univers d'approvisionnement. C'est à ce titre que la Place est valorisée comme lieu, comme moment, comme tissu conjonctif, et c'est à ce titre qu'elle prend sens, à l'échelle de la ville et de l'agglomération : comme manifestation urbaine de ritualités minoritaires, œuvrant comme une des modalités contemporaines de réorganisation des villes comme espaces et sociétés, quoiqu'elles en disent et même si elles les dévalorisent.

\section{BIBLIOGRAPHIE}

AGIER Michel (2002) L'invention de la ville, Paris, Édition des archives contemporaines, 176 p.

Annales de la Recherche Urbaine (1993) n59-60, Mobilités.

ARIESE (1987) Commerces et entrepreneurs ethniques, Université Lyon 2, 104 p.

BATTEGAY Alain (1992) Approches urbaines de l'immigration : la question des territoires ethniques dans les villes françaises, Revue Européenne des Migrations internationales, Vol 8, $\mathrm{n}^{\circ} 2$, pp. 83-100.

BATTEGAY Alain (1996) Le migrant acteur, la migration comme activité, in Michel Péraldi et Éliane PERRIN Éds., Réseaux productifs et territoires urbains, Toulouse, Presses Universitaire du Mirail, pp. 55-70.

BEGAG Azouz (1991) La ville des autres, la famille immigrée et l'espace urbain, Lyon, PUL.

BENVENISTE Annie (1989) Du Bosphore à la Roquette, la communauté judéo-espagnole à Paris (1914-1940), Paris, L'Harmattan.

BONNET Michel, DESJEUX Dominique (2000) Les territoires de la mobilité, Paris, PUF. 
BORDREUIL Jean-Samuel (1994) Centralité urbaine, ville, mobilités, Le Courrier du CNRS, nº 81. BOTTIN Jacques, CALABI Donatella (1999) Les étrangers dans la ville, Minorités et espace urbain du bas Moyen Age à l'époque moderne, Paris, Éditions de la Maison des Sciences de l'Homme, 486 p.

BRISEBARRE Anne-Marie (1998) La fête du mouton, un sacrifice musulman dans l'espace urbain, Paris, CNRS Éditions, $351 \mathrm{p}$.

BRUNEAU Michel (1994) Espaces et territoires de diasporas, L’Espace géographique, $\mathrm{n}^{\circ}$ 1, pp. 5-17.

Cahiers du GREMMO (1996), Territoires et mobilités, $\mathrm{n}^{\circ} 5$.

DE CERTEAU Michel (1978) L'invention du quotidien, Paris, UGE, 10/18, 2 vol.

DE RUDDER Véronique, GUILLON Michèle (1987) Du marché d'Aligre à l'îlot Chalon, Paris, L'Harmattan.

DI MÉO Guy (1997) Les territoires du quotidien, Paris, L'Harmattan, Géographie sociale.

DURAND Marie-Françoise (1994) Entre territoires et réseaux, in Bertrand Badie et Catherine Withol de Wenden (dir.), le Défi migratoire, Paris, Presses de la Fondation nationale des Sciences Politiques.

DURAND Marie-Françoise, LEVY Jacques, RETAILLÉ Denis (1993) Le monde, espace et système, Paris, Presses de la Fondation nationale des Sciences Politiques et Dalloz.

GOFFMAN Erving (1991) Les cadres de l'expérience, Paris, Minuit.

GREEN Nancy (1998) Du Sentier à la $7^{e}$ Avenue La confection et les immigrés Paris New York 1880-1980, Paris, Seuil, coll. L'univers historique.

GUILLON Michèle, TABOADA-LEONETTI Isabelle (1986) Le triangle de Choisy, un quartier chinois à Paris, Paris, L'Harmattan-Ciemi.

GUILMOTO (1990) Les immigrés dans le tissu urbain : Berlin, Londres, Paris, Plan Urbain.

HALBWACHS Maurice (1960) La mémoire collective, Paris, PUF.

HANNERZ Ulf (1984) Explorer la ville, Paris, Minuit.

HANNERZ Ulf (1992) Cultural complexity, studies in the social organisation of meanings, Columbia, Columbia University Press.

KHELLIL Mohand (1980) L'exil kabyle, Paris, L'Harmattan.

MA MUNG Emmanuel (1992) Dispositif économique et ressources spatiales : éléments d'une économie de diaspora, Revue Européenne des Migrations Internationales, vol 8.

MA MUNG Emmanuel (1994) L'entreprenariat ethnique en France, Sociologie du Travail, $n^{\circ} 2$, pp. 185-201.

MA MUNG Emmanuel (1996) Non-lieu et utopie : la diaspora chinoise et le territoire, in Michel Bruneau Éd., Les diasporas, Montpellier, Reclus, pp. 163-173.

MA MUNG Emmanuel (1999) Territorialisation marchande et négociation des identités : les Chinois de Paris, Espace et société, pp. 146-162.

METRAL Jean (coord.) (2000) Cultures en ville, L'aube, 254 p.

MONTULET (1998) Les enjeux spatio-temporels du social, Paris, L'Harmattan.

MOREL Bernard (1991) La recomposition marseillaise, Vingtième siècle, Octobre-Décembre.

OFFNER Jean-Marc, PUMAIN Danièle (1996) Réseaux et territoires, L’Aube. 
PERALDI Michel, PERRIN Evelyne Éds., (1996) Réseaux productifs et territoires urbains, Toulouse, Presses Universitaire du Mirail, 386 pages.

PERALDI Michel (dir.) (2001) Cabas et containers, activités marchandes informelles et réseaux migrants transfrontaliers, Paris, Maisonneuve et Larose, $361 \mathrm{p}$.

PIOLLE Xavier (1990) Mobilité, identités, territoires, Revue de Géographie de Lyon, vol 65, nº 3.

PORTES Alejandro (1999) La mondialisation par le bas. L'émergence de communautés transnationales, Actes de la Recherche en Sciences sociales, ${ }^{\circ}$ 129, pp. 15-25.

RAULIN Anne (1988) Espaces marchands et concentrations minoritaires. La Petite Asie de Paris, Cahiers Internationaux de Sociologie, vol LXXXV.

RAULIN Anne (1986) Mise en scène des commerces maghrébins parisiens, Terrain, $\mathrm{n}^{\circ} 7$.

RAULIN Anne (2000) L'ethnique est quotidien. Diasporas, marchés et cultures métropolitaines, Paris, L'Harmattan.

ROY Olivier (2002) L'islam mondialisé, Paris, Seuil, 210 p.

SASSEN Saskia (1994) L'ethnicité et l'espace dans la ville globale, les Annales de la Recherche urbaine, $\mathrm{n}^{\circ}$ 64, pp. 90-95.

SIMON Gildas (1979) L'espace des travailleurs tunisiens en France, Structures et fonction d'un champ migratoire international, Thèse d'état de géographie, Université de Poitiers.

SIMON Gildas (1995) Géodynamique des migrations internationales dans le monde, Paris, PUF, 429 p.

TARRIUS Alain (1988) L'aménagement à contretemps : nouveaux territoires immigrés à Marseille et à Tunis, Paris, L'Harmattan, 185 p.

TARRIUS Alain (1989) Anthropologie du mouvement, Caen, Paradigme, 176 p.

TARRIUS Alain (1992) Les fourmis d'Europe, migrants riches, migrants pauvres et nouvelles villes internationales, Paris, L'harmattan, $206 \mathrm{p}$.

TARRIUS Alain (1995) Arabes de France dans l'économie mondiale souterraine, Éditions de L'aube.

TARRIUS Alain (2002) La mondialisation par le bas, Paris, Balland, 169 p.

TOUBON Jean-Claude, MESSAMAH Khelifa (1991) Centralité immigrée, Le quartier de la Goutte d'Or, Paris, L'Harmattan-Ciemi, 2 vol.

VELTZ Pierre (1996) Mondialisation, ville et territoires : une économie d'archipel, Paris, PUF.

Sur la Place du Pont

BAHFIR Layachi (1999) L'appropriation de la Place du Pont par les vieux immigrés Maghrébins, mémoire de Maîtrise en Sociologie, Université Lyon 2.

BEGAG Azouz (1997) Place du Pont ou la médina de Lyon, Autrement, Série monde n 96, 83 p.

BELBAHRI Abdelkader (1988) Réseaux sociaux, centralités urbaines et stratégies d'implantation commerciale, La place du Pont à Lyon, Rapport pour le Plan Urbain, $100 \mathrm{p}$.

BERTHET Jean-Marc (1992) La Restructuration d'un espace public : l'exemple de la Place Gabriel Péri à Lyon, mémoire de DEA, université Lyon 2.

DEFOSSE Jacques (1987) Le rôle des structures commerciales dans la formation d'un quartier à dominante immigrée entre la place Gabriel Péri et la Part-Dieu, Université Lyon 2, 125 p.

KLEINCLAUSZ A. (1925) Lyon des origines à nos jours. La formation de la cité, Paris, Masson, $432 \mathrm{p}$. 
Les cahiers du Millénaire (1999) L'agglomération lyonnaise en perspectives, l'exemple du quartier Moncey, $\mathrm{n}^{\circ} 14,62 \mathrm{p}$.

MASSARD-GUILBAUD G. (1995) Des Algériens à Lyon, de la Grande guerre au Front populaire, Paris, L'Harmattan-Ciemi.

RAUTENBERG Michel (1989) Formes et pratiques du commerce ethnique autour de la Place du Pont à Lyon, Le Monde Alpin et Rhodanien, pp. 109-120.

\section{NOTES}

1. Cette recherche a bénéficié de l'appui du Plan Urbain Construction et Architecture du ministère de l'Équipement, dans le cadre du programme « Mobilités et territoires urbains ». Cet article reprend des observations et des analyses conduites en collaboration avec Ahmed Benbouzid, Ahmed Boubeker, Farida Hasnaoui.

\section{RÉSUMÉS}

Le quartier de la Place du Pont est un quartier populaire et immigré du centre-ville de Lyon. Ce morceau de ville est de peu de poids à l'échelle de l'agglomération, en habitants et en superficie, et fait l'objet de procédures lourdes et disputées de renouvellement urbain depuis plus de 10 ans. Mais, la Place du Pont désigne, pour les populations notamment maghrébines qui la fréquentent, un lieu-relais de la ville, un carrefour de réseaux de sociabilités et d'affaires, un espace de ressources et d'approvisionnement.

Aujourd'hui, la Place du Pont mobilise des clientèles habitant l'ensemble de l'agglomération, de l'aire urbaine, de la région et au-delà. Les offres commerciales qu'elle propose tendent à concentrer des services, requis par la circulation d'informations, d'hommes et de marchandises, ainsi que des produits pour des « mobilités culturelles sur place». La Place du Pont apparaît ainsi comme une vitrine où s'exposent et se mettent à l'épreuve de nouveaux produits et de nouveaux services adaptés à des univers d'approvisionnement qui ont des dimensions commerciales et culturelles, économiques et sociales, et comme un espace de ressources pour des populations dont les manières de vivre en migration se diversifient et se recomposent. Alors même que son destin urbain est incertain, la Place du Pont s'affirme comme un lieu et un moment urbain de ritualités minoritaires.

An immigrant market centre in transition: the Place du Pont, Lyon. The Place du Pont is a working-class immigrant neighbourhood in the centre of Lyon. This sector of the city, though relatively unimportant in terms of the population and area of the city, has been the object of extensive and controversial urban renewal policies for a decade. But for those who frequent the Place du Pont, the place is a key area of the city, a centre of social and commercial networks, a centre of resources and provisions.

Today the Place du Pont is host to clienteles from all over city, the urban area, the region and beyond. The business opportunities and services it offers make for a concentration of resources that are needed for the circulation of information, people and commodities, as well as for products for «localized cultural circulation». The Place du Pont is thus a showcase for new 
products and services corresponding to certain types of supplies in their cultural, economic and social aspects, and as an area of resources for groups whose mode of life has been changed and reorganized through migration. While its urban future may be uncertain, the Place du Pont has already established itself as an urban time-space for minority social patterns.

Las recomposiciones de una zona comercial immigrante : la Place du Pont de Lyon. El barrio de la Place du Pont es un barrio popular y de inmigración del centro de la ciudad de Lyon. En lo que a habitantes y a superficie se refiere, esta zona de la ciudad ostenta poco peso en el seno de la aglomeración $\mathrm{y}$, desde hace más de diez años, su renovación urbana ha dado lugar a procedimientos complicados y disputados. Pero, para la población, principalmente magrebí, que frecuenta el barrio, ésta constituye un lugar de referencia dentro de la ciudad, un cruce de redes de sociabilidad y de negocios, un espacio de recursos y de abastecimiento.

Hoy en día, la Place du Pont atrae a clientelas de toda la aglomeración, del área urbana, de la región y de más allá. Las ofertas comerciales que propone se centran en servicios directamente ligados a la circulación de informaciones de hombres como de mercancías, y en productos destinados a las "movilidades culturales in situ". La Place du Pont es una vitrina en la que se exponen y se prueban nuevos productos y servicios adaptados a universos de aprovisionamiento dotados de dimensiones comerciales y culturales, económicas y sociales. La Place du Pont es, al mismo tiempo, un espacio de recursos para las poblaciones cuya manera de vivir la inmigración se diversifica y se recompone. En un momento en el que reina la incertidumbre con respecto al destino urbano del barrio, éste se afirma como un lugar y un momento urbano de rituales minoritarios.

\section{AUTEUR}

\section{ALAIN BATTEGAY}

Sociologue CNRS, Groupe de Recherches sur la socialisation UMR 5040/ARIESE, Université Lumière, Lyon II, 5 avenue Pierre Mendès France, 69500 Bron. 